Forum $2022 \cdot 37: 1-2$

https://doi.org/10.1007/s12312-021-01012-0

(c) Springer Medizin Verlag $\mathrm{GmbH}$, ein Teil von Springer Nature 2021

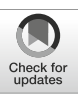

\title{
Schnittstellen zwischen Innovation und Versorgung: interdisziplinäre Zusammenarbeit ausgestalten
}

Anja Mehnert-Theuerkauf

Abt. für Medizinische Psychologie und Medizinische Soziologie, Universitätsklinikum Leipzig, Leipzig, Deutschland

Liebe Kolleginnen, liebe Kollegen,

der Deutsche Krebskongress (DKK) 2022 greift unter der Federführung von Prof. Dr. Michael Ghadimi ein hoch relevantes Thema der Krebsmedizin auf: Schnittstellen zwischen Innovation und Versorgung. Dabei geht es v.a. um die Ausgestaltung der Prozesse, wie Erfolg versprechende Ergebnisse der Grundlagenforschung in wirksame Therapien überführt werden können. Bessere Forschung, Translation und Versorgung in der Onkologie erfordern aber auch, dass die Zusammenarbeit der Fachkräfte im Gesundheitswesen an den Schnittstellen weiterentwickelt wird, mit dem gemeinsamen Ziel, Patienten zu einem längeren und gesünderen Leben zu verhelfen. Dies gilt für Grundlagenforscher und Kliniker ebenso wie für Spezialisten unterschiedlicher Disziplinen und Patientenvertreter.

Idealerweise erfolgt interdisziplinäre Zusammenarbeit bei komplexen Krankheiten wie Krebs in allen Phasen von der Prävention und Vorsorge, über die Therapie bis hin zur Rehabilitation und Nachsorge - und setzt ein integratives Verständnis der Krankheitsentstehung, Behandlung und der Krankheitsfolgen voraus. Der Gedanke ist nicht neu und wurde bereits in dem von Engel formulierten „biopsychosozialen Modell von Gesundheit und Krankheit" [1] aufgegriffen. Dieser systemtheoretische Ansatz postuliert, dass biologische, psychische und soziale Prozesse auf verschiedenen Ebenen miteinander verbunden sind und sich gegenseitig beeinflussen. Er ist damit für die Entstehung, die Aufrechterhaltung und den Verlauf von Krankheiten von zentraler Bedeutung und unterstreicht den Wert einer engen Vernetzung und Kooperation in Forschung, Translation und Versorgung, da bessere Ergebnisse für den Patienten und sein familiäres Umfeld erzielt werden, wenn Spezialisten interdisziplinär zusammenarbeiten.

Und, viele Fachkräfte im Gesundheitswesen wollen - auch sektorenübergreifend - zusammenarbeiten. Warum gelingt dies im Alltag häufig aber nur bedingt? Alle Berufsgruppen haben kaum Zeit, um mehr zu tun, als sie ohnehin schon tun. Zusammenarbeit kann bedeuten, dass man gemeinsam im Tumorboard sitzt oder sich eine elektronische Patientenakte ansieht; in vielen Fällen ist jedoch deutlich mehr erforderlich, um sicherzustellen, dass Menschen als Team effizient zusammenarbeiten.

\section{») Die Ausgestaltung der Kommunikationskultur ist ein Schlüsselelement erfolgreicher Zusammenarbeit}

Was macht erfolgreiche interdisziplinäre Zusammenarbeit aus? Die Ausgestaltung der Kommunikationskultur ist ein Schlüsselelement. Sich gemeinsame Ziele zu setzen und diese in regelmäßigen Abständen zu evaluieren, gehört ebenso dazu wie eine klare Verantwortungs- und Rollenaufteilung. Jedes Teammitglied hat im Idealfall ein Bewusstsein dafür, welche eigenen spezifischen Fähigkeiten in die Arbeit eingebracht werden und was die Experti- 


\section{Editorial}

se der Kollegen ausmacht. Die Aufteilung von Aufgaben und die Abgrenzung von Verantwortlichkeiten sollten transparent kommuniziert und gegenseitig respektiert werden. Schließlich sind ein vertrauensvoller Umgang miteinander sowie die Bereitschaft wichtig, im Falle von Konflikten Verantwortung für die Problemlösung und die Wiederherstellung einer gelungenen Gesprächs- und Kooperationskultur zu übernehmen. Wir alle müssen effizientere Wege finden, die Zusammenarbeit an Schnittstellen mit dem Ziel zu optimieren, bessere Behandlungsergebnisse für unsere Patienten zu erreichen.

Ich wünsche Ihnen viel Freude und neue Erkenntnisse beim Lesen der anregenden Beiträge dieses Themenheftes rund um den DKK 2022.

Ihre

Anja Mehnert-Theuerkauf

Korrespondenzadresse

Prof. Dr. Anja Mehnert-Theuerkauf Abt. für Medizinische Psychologie und Medizinische Soziologie, Universitätsklinikum Leipzig

Philipp-Rosenthal-Straße 55, 04103 Leipzig,

Deutschland

anja.mehnert@medizin.uni-leipzig.de

Interessenkonflikt. A. Mehnert-Theuerkauf gibt an, dass kein Interessenkonflikt besteht.

\section{Literatur}

1. Engel GL (1977) The need for a new model: a challenge for biomedicine. Science 1296:129-137

\section{Deutscher Krebskongress - Neuer Termin \\ „Schnittstellen zwischen Innovation und Versorgung"}

Der Deutsche Krebskongress ist verschoben und findet vom 13.-16. November 2022 im City Cube in Berlin statt.

\section{PRÄSENZKONGRESS}

Der Kongress ist weiterhin als Präsenzveranstaltung geplant. Vorhaben und Pläne - so haben wir allerdings auch alle gelernt - schmiedet man derzeit mit ungewissem Ausgang. Die DKG freut sich deshalb über Ihre Bereitschaft, flexibel auf alle Entwicklungen vor dem Kongress und während des Kongresses zu reagieren.

Alle Infos rund um den Kongress finden Sie hier:

https://www.deutscher-krebskongress.de

In diesem Heft finden Sie die „Hot Topics DKK 2022: Teil 1 - Vorschau“. Weitere Themen zum Kongress folgen in Ausgabe 5/2022, die auch auf dem Kongress ausgelegt wird.

Wir freuen uns auf ein persönliches Wiedersehen oder Kennenlernen!

Ihre Redaktion der Zeitschrift „Forum“

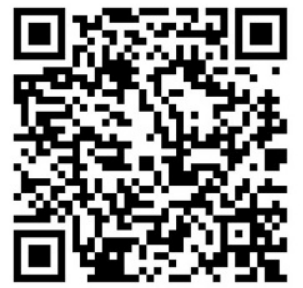

Hier geht's mit dem Smartphone direkt zur Kongresshomepage 